\title{
New developments for dual methods in lattice field theory at non-zero density
}

\author{
Christof Gattringer * \\ Karl-Franzens-Universität, 8010 Graz, Austria \\ E-mail: christof.gattringer@uni-graz.at
}

\begin{abstract}
In recent years the complex action problem of lattice field theory at finite density was overcome for several system by mapping them to dual variables (flux lines and surfaces). We illustrate this mapping for the case of the U(1) gauge Higgs system and present some representative physics results for this model. Conceptual challenges such as spectroscopy in the dual approach, as well as algorithmic developments are discussed and related ideas for systems with fermions are addressed. Models where the dual variables approach solves the complex action problem can serve as reference systems for other approaches to finite density lattice field theory and we discuss some examples. Finally we address the strategy of a partial dualization in certain limits, e.g., for strong coupling and large mass.
\end{abstract}

31st International Symposium on Lattice Field Theory - LATTICE 2013

July 29 - August 3, 2013

Mainz, Germany

*This work has been partly supported by the Austrian Science Fund FWF, DK Hadrons in Vacuum, Nuclei, and Stars (FWF DK W1203-N16), the Research Executive Agency (REA) of the European Union under Grant Agreement number PITN-GA-2009-238353 (ITN STRONGnet), by ERC Hadron Physics 2, by DFG TR55, "Hadron Properties from Lattice QCD” and by the Austrian Science Fund FWF, Grant. Nr. I 1452-N27. 


\section{Introductory remarks}

Numerical simulations of lattice field theories at finite density ${ }^{1}$ have to deal with the "complex action problem": For finite chemical potential $\mu$ the action $S$ becomes complex and the Boltzmann factor $e^{-S}$ can no longer be used as a weight factor in a Monte Carlo simulation. The complex action problem appears for both, bosonic and fermionic systems - for the latter the fermion determinant is complex for non-zero $\mu$. The appearance of a complex action at finite $\mu$ is not a lattice artifact but a genuine feature of many quantum field theories at finite chemical potential.

Many ideas to overcome the complex action problem have been discussed over the years and were covered in reviews at the annual lattice conferences and other occasions [1]-[10]: Reweighting techniques, series expansions, analytic continuation, stochastic methods, density of state techniques, canonical simulations and other approaches were tested and helped to understand aspects of QCD and other lattice field theories at finite density. On the other hand it is also well known that many of these approaches have clear limitations for the values of the chemical potential they can reach and thus can only be considered in a limited range of parameters ${ }^{2}$.

A completely different approach is the idea of trying to find a parametrization of the partition sum in terms of new variables, such that in the transformed partition sum only real and positive contributions appear also at finite chemical potential $\mu$. Having a reformulation with only real and positive contributions then implies that suitably adapted Monte Carlo algorithms can be used to simulate the system at arbitrary $\mu$ and the complex action problem is solved.

Several different strategies were followed for finding suitable parametrizations, and here we will often refer to the new representation used as "dual representation" or "dual variables", although often it is not a conventional duality transformation that has been used for rewriting the system. In recent years dual representations were found for a wide range of models, to a large extent bosonic models with abelian symmetry [32]-[71], but also some non-abelian cases were studied, and with related methods also some fermionic systems were successfully treated [74]-[98].

For many forms of dual representations matter fields assume the form of oriented loops on the lattice and gauge fields turn into surfaces made of plaquettes that can either be closed surfaces or bounded by matter flux ${ }^{3}$. These dual variables are subject to constraints, i.e., for the matter loops the flux is conserved at each site of the lattice, and for the gauge fields a similar constraint on the links ensures that the gauge surfaces are closed or bounded by matter flux. Thus for a successful Monte Carlo simulation one needs to find algorithms that take into account these constraints and generate only configurations that are admissible, i.e., obey all the constraints. For systems with only fluxes, the Prokof'ev - Svistunov worm algorithm [99] and generalizations of it solve this issue. For systems with gauge surfaces local dual updates were used initially [50, 51, 52], but more recently generalizations of the worm idea to surfaces were implemented successfully [53]-[60].

An important aspect of many dual representations is the fact, that not all observables can be rewritten in a trivial way to the dual representation. Straightforward are bulk observables which are obtained as derivatives of the free energy with respect to the parameters of the theory. These

\footnotetext{
${ }^{1}$ Up to a few exceptions such as QCD with gauge group SU(2).

${ }^{2} \mathrm{New}$ developments have been presented for stochastic approaches [1], [11]-[31] and it will be interesting to see how far they reach when realistic models and system sizes are considered.

${ }^{3}$ In the next section we show this explicitly for the U(1) gauge-Higgs system.
} 
derivatives can be evaluated also in the dual representation and the bulk observables assume the form of moments of suitable averages of dual variables. More complicated are $n$-point functions, which are, e.g., needed for spectroscopy calculations. For these one usually introduces source terms, which, however, change the form of the dual representation: The source terms introduce local contributions to the matter loops, i.e., the constraints for the matter flux are violated at the points where the sources are activated. The resulting representation can be viewed as a more general dual form with defects at some of the sites. Suitable generalizations of the worm algorithm were discussed [61] and the feasibility of dual spectroscopy at finite $\mu$ was demonstrated [62, 63].

Let us conclude these introductory remarks with pointing out possible applications of models where the complex action problem has been solved completely with dual techniques. A first application is of course the study of interesting non-perturbative phenomena in finite density field theories. An important example is the so-called Silver Blaze phenomenon [111], which is the fact that as long as the chemical potential $\mu$ is smaller than the mass $m_{0}$ of the lightest excitation, physics is independent of $\mu$, and only at $\mu=m_{0}$ condensation sets in. The Silver Blaze phenomenon was studied in various theories recently $[25,26,29,64,71,104,106,109,110]$. Besides ab-initio studies of density related phenomena in quantum field theories, the systems that can be solved with dual methods may also serve as reference models to test other approaches to finite density on the lattice. Examples where stochastic methods were compared to dual results can be found in [1, 19, 27, 29], and tests of series expansions in $\mu$ were presented in [47, 48].

\section{Dual representation for the U(1) gauge Higgs system}

In this section we discuss the mapping of the $\mathrm{U}(1)$ gauge Higgs model to a dual representation. This example is characteristic for similar mappings in other theories with matter and gauge fields and illustrates the essential steps, as well as the typical structure of the dual representation.

The degrees of freedom are gauge fields $U_{x, v} \in \mathrm{U}(1)$ on the links of the lattice and a charged scalar Higgs field $\phi_{x} \in \mathbb{C}$, attached to the sites. We consider a 4-dimensional lattice with volume $V_{4}=N_{s}^{3} \times N_{t}$ and periodic boundary conditions for both fields. The total action is a sum of gauge and matter parts, $S=S_{G}+S_{M}$. For the gauge action we use the standard Wilson form,

$$
S_{G}=-\frac{\beta}{2} \sum_{x} \sum_{v<\rho}\left[U_{x, v \rho}+U_{x, v \rho}^{*}\right],
$$

where $U_{x, v \rho}=U_{x, v} U_{x+\hat{v}, \rho} U_{x+\hat{\rho}, v}^{*} U_{x, \rho}^{*}$ and $\beta$ is the inverse gauge coupling. The action for the matter fields is given by

$$
S_{M}=\sum_{x}\left[M^{2}\left|\phi_{x}\right|^{2}+\lambda\left|\phi_{x}\right|^{4}-\sum_{v}\left(e^{-\mu \delta_{v 4}} \phi_{x}^{*} U_{x, v} \phi_{x+\hat{v}}+e^{\mu \delta_{v 4}} \phi_{x} U_{x, v}^{*} \phi_{x+\hat{v}}^{*}\right)\right] .
$$

The parameter $M^{2}$ denotes $8+m^{2}$, where $m$ is the bare mass parameter and $\lambda$ is the quartic coupling. A non-zero chemical potential $\mu$ gives a different weight to forward and backward temporal hops (the temporal direction is the 4-direction and the Kronecker delta $\delta_{v 4}$ switches on the chemical potential terms only for $v=4$ ) and it is obvious that for $\mu \neq 0$ the action becomes complex. In the standard representation the gauge Higgs model thus has a complex action problem. 
The partition sum $Z=\int D[U] D[\phi] e^{-S_{G}-S_{M}}$ is given as an integral over all field configurations. For the Higgs field the measure is a product over all lattice points $x$, and we use polar coordinates $\phi_{x}=r_{x} e^{i \theta_{x}}$ for integrating each $\phi_{x}$ in the complex plane. The $\mathrm{U}(1)$ gauge variables $U_{x, v}=e^{i \varphi_{x, v}}$ at each link are integrated over the unit circle such that the path integral reads

$$
Z=\int D[U] D[\phi] e^{-S_{G}-S_{M}}=\left(\prod_{x, v} \int_{-\pi}^{\pi} \frac{d \varphi_{x, v}}{2 \pi}\right)\left(\prod_{x} \int_{-\pi}^{\pi} \frac{d \theta_{x}}{2 \pi} \int_{0}^{\infty} d r_{x} r_{x}\right) e^{-S_{G}-S_{H}} .
$$

We begin the mapping to the dual representation with the Higgs part of the problem and define the partition sum of the Higgs system in a gauge background as

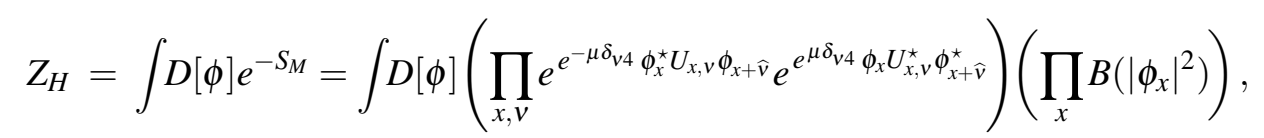

where we have reorganized the nearest neighbor terms and $B\left(r^{2}\right) \equiv \exp \left(-M^{2} r^{2}-\lambda r^{4}\right)$. The Boltzmann factors for the nearest neighbor terms (use $U_{x, v}{ }^{\star}=U_{x, v}{ }^{-1}$ ) are now expanded,

$$
\begin{aligned}
& \prod_{x, v} \exp \left(e^{-\mu \delta_{v 4}} \phi_{x}^{\star} U_{x, v} \phi_{x+\widehat{v}}\right) \exp \left(e^{\mu \delta_{v 4}} \phi_{x} U_{x, v}^{\star} \phi_{x+\widehat{v}}^{\star}\right)= \\
& \left(\prod_{x, v} \sum_{n_{x, v}=0}^{\infty} \sum_{\bar{n}_{x, v}=0}^{\infty}\right)\left(\prod_{x, v} \frac{e^{-\mu n_{x, 4} \delta_{v 4}} e^{\mu \bar{n}_{x, 4} \delta_{v 4}} U_{x, v}^{n_{x, v}} U_{x, v}^{\star} \bar{n}_{x, v}}{n_{x, v} ! \bar{n}_{x, v} !}\right)\left(\prod_{x, v}\left(\phi_{x}^{\star} \phi_{x+\widehat{v}}\right)^{n_{x, v}}\left(\phi_{x} \phi_{x+\widehat{v}}^{\star}\right)^{\bar{n}_{x, v}}\right)= \\
& \sum_{\{n, \bar{n}\}}\left(\prod_{x, v} \frac{U_{x, v}^{n_{x, v}-\bar{n}_{x, v}}}{n_{x, v} ! \bar{n}_{x, v} !}\right)\left(\prod_{x} e^{-\mu\left(n_{x, 4}-\bar{n}_{x, 4}\right)}\right)\left(\phi_{x}^{\star \sum_{v}\left[n_{x, v}+\bar{n}_{x-\widehat{v}, v}\right]} \phi_{x}{ }^{\sum_{v}\left[\bar{n}_{x, v}+n_{x-\widehat{v}, v}\right]}\right),
\end{aligned}
$$

where the expansion variables $n_{x, v}$ and $\bar{n}_{x, v}$ are non-negative integers attached to the links of the lattice. By $\sum_{\{n, \bar{n}\}}$ we denote the sum over all configurations of the expansion variables $n_{x, v}, \bar{n}_{x, v} \in$ $[0, \infty)$. The partition sum of the Higgs field now reads

$$
\begin{aligned}
Z_{H}=\sum_{\{n, \bar{n}\}}( & \left.\prod_{x, v} \frac{U_{x, v}^{n_{x, v}-\bar{n}_{x, v}}}{n_{x, v} ! \bar{n}_{x, v} !}\right)\left(\prod_{x} e^{-\mu\left(n_{x, 4}-\bar{n}_{x, 4}\right)}\right)\left(\prod_{x} \int_{-\pi}^{\pi} \frac{d \theta_{x}}{2 \pi} e^{-i \theta_{x} \sum_{v}\left[n_{x, v}-\bar{n}_{x, v}-\left(n_{x-\widehat{v}, v}-\bar{n}_{x-\widehat{v}, v}\right)\right]}\right) \\
& \times\left(\prod_{x} \int_{0}^{\infty} d r_{x} r_{x}^{1+\sum_{v}\left[n_{x, v}+n_{x-\widehat{v}, v}+\bar{n}_{x, v}+\bar{n}_{x-\widehat{v}, v}\right]} B\left(r_{x}^{2}\right)\right)
\end{aligned}
$$

The integrals over the phase give rise to Kronecker deltas, which for notational convenience here we write as $\delta(n)$. For the integrals over the modulus we introduce the abbreviation $P(n)=$ $\int_{0}^{\infty} d r r^{n+1} B\left(r^{2}\right)$. The $P(n)$ can be computed numerically and are pre-stored for determining the acceptance in the Metropolis steps of the Monte Carlo. The partition sum for the Higgs field is

$$
\begin{aligned}
Z_{H}=\sum_{\{n, \bar{n}\}}( & \left.\prod_{x, v} \frac{U_{x, v}^{n_{x, v}-\bar{n}_{x, v}}}{n_{x, v} ! \bar{n}_{x, v} !}\right)\left(\prod_{x} e^{-\mu\left(n_{x, 4}-\bar{n}_{x, 4}\right)}\right)\left(\prod_{x} \delta\left(\sum_{v}\left[n_{x, v}-\bar{n}_{x, v}-\left(n_{x-\widehat{v}, v}-\bar{n}_{x-\widehat{v}, v}\right)\right]\right)\right) \\
& \times\left(\prod_{x} P\left(\sum_{v}\left[n_{x, v}+\bar{n}_{x, v}+n_{x-\widehat{v}, v}+\bar{n}_{x-\widehat{v}, v}\right]\right)\right)
\end{aligned}
$$

In this form the Higgs fields are integrated out and the partition sum is a sum over configurations of the $n$ and $\bar{n}$. The allowed configurations of the $n$ and $\bar{n}$ are subject to local constraints at each 
site $x$ enforced by the Kronecker deltas, i.e., at each site $x$ the variables must obey $\sum_{v}\left[n_{x, v}-\bar{n}_{x, v}-\right.$ $\left.\left(n_{x-\widehat{v}, v}-\bar{n}_{x-\widehat{v}, v}\right)\right]=0$. We stress that in the new form (2.7) the chemical potential enters only via the real factors $e^{-\mu\left(n_{x, 4}-\bar{n}_{x, 4}\right)}$ which is the key step for solving the complex action problem.

The structure of the constraints can be simplified by introducing new variables $l_{x, v} \in(-\infty, \infty)$ and $\bar{l}_{x, v} \in[0, \infty)$, which are related to the old variables by $n_{x, v}-\bar{n}_{x, v}=l_{x, v}$ and $n_{x, v}+\bar{n}_{x, v}=\left|l_{x, v}\right|+$ $2 \bar{l}_{x, v}$, and the sum over all configurations of the $n, \bar{n}$ variables can be replaced by a sum over $l$ - and $\bar{l}$-configurations. The partition sum turns into

$$
\begin{aligned}
Z_{H}=\sum_{\{l, \bar{l}\}}( & \left.\prod_{x, v} \frac{U_{x, v}^{l_{x, v}}}{\left(\left|l_{x, v}\right|+\bar{l}_{x, v}\right) ! \bar{l}_{x, v} !}\right)\left(\prod_{x} e^{-\mu l_{x, 4}}\right)\left(\prod_{x} \delta\left(\sum_{v}\left[l_{x, v}-l_{x-\widehat{v}, v}\right]\right)\right) \\
& \times\left(\prod_{x} P\left(\sum_{v}\left[\left|l_{x, v}\right|+\left|l_{x-\widehat{v}, v}\right|+2\left(\bar{l}_{x, v}+\bar{l}_{x-\widehat{v}, v}\right)\right]\right)\right) .
\end{aligned}
$$

In the final form (2.8) of the Higgs field partition sum, which we now refer to as dual representation, the constraints no longer mix the two types of flux variables. Obviously only the $l$-fluxes are subject to conservation of flux at each site $x$, i.e., they must obey $\sum_{v}\left[l_{x, v}-l_{x-\widehat{v}, v}\right]=0$ for all $x$.

Having mapped the Higgs field partition sum to the flux form (2.8) we now apply similar steps to the gauge fields to obtain the dual representation of the full partition sum (2.3). We write the full partition sum as $Z=\int D[U] e^{-S_{G}} Z_{H}$ and find

$$
\begin{aligned}
Z= & \sum_{\{l, \bar{l}\}}\left(\prod_{x, v} \frac{1}{\left(\left|l_{x, v}\right|+\bar{l}_{x, v}\right) ! \bar{l}_{x, v} !}\right)\left(\prod_{x} e^{-\mu l_{x, 4}}\right)\left(\prod_{x} \delta\left(\sum_{v}\left[l_{x, v}-l_{x-\widehat{v}, v}\right]\right)\right) \\
& \times\left(\prod_{x} P\left(\sum_{v}\left[\left|l_{x, v}\right|+\left|l_{x-\widehat{v}, v}\right|+2\left(\bar{l}_{x, v}+\bar{l}_{x-\widehat{v}, v}\right)\right]\right)\right) Z_{G}[l],
\end{aligned}
$$

where we have interchanged the sum over the flux configurations and the integral over the gauge fields. The gauge field partition sum with link insertions according to a flux configuration $l$ is defined as $Z_{G}[l]=\int D[U] e^{-S_{G}} \prod_{x, v} U_{x, v}^{l_{x, v}}$. The gauge action $S_{G}$ as defined in (2.1) is a sum over plaquettes. We thus may write the Boltzmann factor $e^{-S_{G}}$ as a product over plaquettes and, as done for the Higgs field, we expand the corresponding exponentials into power series:

$$
\begin{aligned}
& \prod_{x, \sigma<\tau} e^{\frac{\beta}{2} U_{x, \sigma \tau}} e^{\frac{\beta}{2} U_{x, \sigma \tau}^{\star}}=\sum_{\{m, \bar{m}\}}\left(\prod_{x, \sigma<\tau} \frac{\left(\frac{\beta}{2}\right)^{m_{x, \sigma \tau}+\bar{m}_{x, \sigma \tau}}}{m_{x, \sigma \tau} ! \bar{m}_{x, \sigma \tau} !} U_{x, \sigma \tau}{ }^{m_{x, \sigma \tau}} U_{x, \sigma \tau}^{\star \bar{m}_{x, \sigma \tau}}\right) \\
& =\sum_{\{m, \bar{m}\}}\left(\prod_{x, \sigma<\tau} \frac{\left(\frac{\beta}{2}\right)^{m_{x, \sigma \tau}+\bar{m}_{x, \sigma \tau}}}{m_{x, \sigma \tau} ! \bar{m}_{x, \sigma \tau} !}\right)\left(\prod_{x, v} U_{x, v} \sum_{v<\alpha}\left[p_{x, v \alpha}-p_{x-\widehat{\alpha}, v \alpha}\right]-\sum_{\alpha<v}\left[p_{x, \alpha v}-p_{x-\widehat{\alpha}, \alpha v}\right]\right) .
\end{aligned}
$$

We introduced the expansion variables $m_{x, \sigma \tau}, \bar{m}_{x, \sigma \tau} \in[0, \infty)$ attached to the plaquettes, and by $\sum_{\{m, \bar{m}\}}$ we denote the sum over all configurations of the expansion variables. In the second step we inserted the explicit expressions for the plaquettes in terms of the link variables, i.e., $U_{x, \sigma \tau}=U_{x, \sigma} U_{x+\widehat{\sigma}, \tau} U_{x+\widehat{\tau}, \sigma}^{\star} U_{x, \tau}^{\star}$, and reorganized the product over powers of links variables. Here we already introduced $m_{x, v \alpha}-\bar{m}_{x, v \alpha}=p_{x, v \alpha}$. We promote $p_{x, v \alpha} \in(-\infty, \infty)$ into new dynamical variables, which together with another set of variables, $q_{x, v \alpha} \in[0, \infty)$, gives the final set of variables we use for the gauge fields. The $p$ and $q$ variables are related to the $m$ and $\bar{m}$ variables via $m_{x, v \alpha}-\bar{m}_{x, v \alpha}=p_{x, v \alpha}$ and $m_{x, v \alpha}+\bar{m}_{x, v \alpha}=\left|p_{x, v \alpha}\right|+2 q_{x, v \alpha}$. We will refer to the variables $p$ as 
plaquette occupation numbers or simply plaquette variables. Using the new variables and inserting the expanded Boltzmann factor (2.10) back into $Z_{G}$ we find

$$
\begin{aligned}
Z_{G}[l] & =\sum_{\{p, q\}}\left(\prod_{x, \sigma<\tau} \frac{\left(\frac{\beta}{2}\right)^{\left|p_{x, \sigma \tau}\right|+2 q_{x, \sigma \tau}}}{\left(\left|p_{x, \sigma \tau}\right|+q_{x, \sigma \tau}\right) ! q_{x, \sigma \tau} !}\right) \\
& \times\left(\prod_{x, v} \int_{-\pi}^{\pi} \frac{d \varphi_{x, v}}{2 \pi} e^{i \varphi_{x, v}\left(\sum_{v<\alpha}\left[p_{x, v \alpha}-p_{x-\widehat{\alpha}, v \alpha}\right]-\sum_{\alpha<v}\left[p_{x, \alpha v}-p_{x-\widehat{\alpha}, \alpha v}\right]+l_{x, v}\right)}\right) .
\end{aligned}
$$

The integrals in the last product are again representations of Kronecker deltas and give rise to constraints that are located at the links of the lattice. The summations over the variables $q_{x, \sigma \tau}$ can be done in closed form using the well known series representation of the modified Bessel functions $\sum_{q=0}^{\infty} \frac{\left(\frac{\beta}{2}\right)^{|p|+2 q}}{(|p|+q) ! q !}=I_{|p|}(\beta)=I_{p}(\beta)$. Thus we end up with the following representation for $Z_{G}$ :

$$
Z_{G}[l]=\sum_{\{p\}}\left(\prod_{x, \sigma<\tau} I_{p_{x, \sigma \tau}}(\beta)\right)\left(\prod_{x, v} \delta\left(\sum_{v<\alpha}\left[p_{x, v \alpha}-p_{x-\widehat{\alpha}, v \alpha}\right]-\sum_{\alpha<v}\left[p_{x, \alpha v}-p_{x-\widehat{\alpha}, \alpha v}\right]+l_{x, v}\right)\right) .
$$

Putting this back into the full partition sum (2.9) we obtain the final result for the dual representation of the partition sum for the U(1) gauge Higgs model:

$$
Z=\sum_{\{\bar{l}, l\}\{p\}} \sum_{M} \mathscr{W}_{M}[\bar{l}, l] \mathscr{W}_{G}[p] \mathscr{C}_{S}[l] \mathscr{C}_{L}[p, l]
$$

The weight factors are

$$
\begin{aligned}
\mathscr{W}_{M}[\bar{l}, l] & =\prod_{x, v} \frac{1}{\left(\left|l_{x, v}\right|+\bar{l}_{x, v}\right) ! \bar{l}_{x, v} !} \exp \left(-\mu \sum_{x} l_{x, 4}\right) \prod_{x} P\left(\sum_{v}\left[\left|l_{x, v}\right|+\left|l_{x-\hat{v}, v}\right|+2\left(\bar{l}_{x, v}+\bar{l}_{x-\hat{v}, v}\right)\right]\right), \\
\mathscr{W}_{G}[p] & =\prod_{x, \rho<v} I_{p_{x, \rho v}}(\beta) .
\end{aligned}
$$

It is obvious that all weight factors are real and positive and thus the complex action problem has vanished in the dual representation. The $l$ and the $p$ variables are subject to constraints given by

$$
\begin{aligned}
\mathscr{C}_{L}[p, l] & =\prod_{x} \prod_{v=1}^{4} \delta\left(\sum_{\rho: v<\rho}\left[p_{x, v \rho}-p_{x-\hat{\rho}, v \rho}\right]-\sum_{\rho: v>\rho}\left[p_{x, \rho v}-p_{x-\hat{\rho}, \rho v}\right]+l_{x, v}\right), \\
\mathscr{C}_{S}[l] & =\prod_{x} \delta\left(\sum_{v=1}^{4}\left[l_{x-\hat{v}, v}-l_{x, v}\right]\right) .
\end{aligned}
$$

The constraints come in two parts: We have constraints $\mathscr{C}_{S}[l]$ for the variables $l$ that are based at the sites and enforce the total $l$-flux (flux for matter fields) to vanish at each site of the lattice. The second type of constraints $\mathscr{C}_{L}[p, l]$ are based on the links and combine $p$ and $l$ variables. They enforce the total flux from matter and from the plaquettes to vanish at each link.

We have already stated that typically the dual representation gives rise to loops for the matter degrees of freedom, and surfaces for the gauge fields, where the latter are either closed surfaces or surfaces bounded by matter flux. This geometrical interpretation is a direct consequence of the constraints and in Fig. 1 we show an example of an admissible dual configuration, i.e., a configuration where all constraints are obeyed. The flux variables $l_{x, v}$ are represented by full red lines 


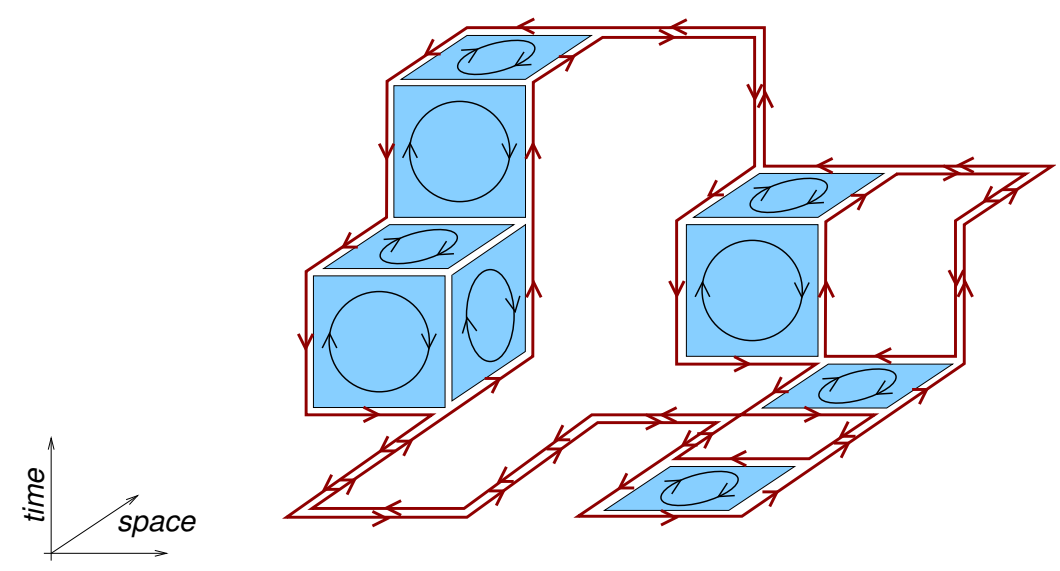

Figure 1: Example of an admissible configuration of the dual variables in the U(1) gauge Higgs system.

attached to the links with arrows indicating the amount of flux, where the arrows for negative values point in negative direction (the example shown in Fig. 1 only has $l_{x, v}= \pm 1$ ). Plaquette variables $p_{x, v \rho}$ are represented by fluxes drawn onto the corresponding plaquettes. The example in Fig. 1 illustrates that at each site the matter flux vanishes, and that at each link the total flux from matter and plaquette variables adds up to zero as well.

It interesting to analyze the term $\exp \left(-\mu \sum_{x} l_{x, 4}\right)$ where the chemical potential couples: In the sum $\sum_{x} l_{x, 4}$ the 4-components of the $l$-flux (which come with positive and negative signs) are added up. Since flux is conserved due to the constraints, this term corresponds to $N_{t}$ times the total net flux of $l$ around the temporal direction. Since $N_{t}$ corresponds to the inverse temperature $\beta$ in lattice units (not to be confused with the inverse gauge coupling here), we can identify the particle number $N$ via $\exp (\mu \beta N)=\exp \left(-\mu \sum_{x} l_{x, 4}\right)$ and find $N=-\sum_{x} l_{x, 4} / N_{t}$.

The fact that the chemical potential couples to the net $l$-flux that winds around the compactified time direction has now to be confronted with the link constraints that have to be obeyed. They imply that on each link the $l$-flux is either directly matched by one with opposite sign, which, however, leads to a cancellation of the corresponding factors $e^{ \pm \mu}$, or compensated by a suitable plaquette value $p_{x, v \rho}$, which, however, only shifts the flux that needs to be compensated to a neighboring link. It is easy to see that for obeying the link constraints one is either forced to a vanishing particle number $N=-\sum_{x} l_{x, 4} / N_{t}=0$, and thus no $\mu$-dependence, or to attaching infinitely many plaquettes to "shift the problem to infinity", which gives rise to a configuration with infinite energy. Obviously the dual representation knows about the neutrality condition of electrodynamics and for having a non-trivial dependence on the chemical potential one needs several flavors with vanishing total charge. The simplest case is to add a second Higgs field with opposite charge, and in this theory physics at finite chemical potential can be studied [59, 60, 70, 71] (see also below).

The construction which we discussed here can be generalized to essentially all abelian gauge Higgs systems - for an example with $\mathbb{Z}_{3}$ symmetry see [49]. The question for the existence of a real dual representation for systems with abelian symmetries was analyzed in a more general setting in $[68,69]$ and the relation to discrete symmetries was clarified. We note that in some special (low dimensional) models exploring discrete symmetries led to an algorithm ("subset method") that overcomes the complex action problem already in the conventional representation [112]-[115]. 


\section{Algorithms}

For a successful Monte Carlo simulation it is mandatory to use an algorithm that produces only admissible configurations of the dual variables, i.e., configurations that obey all the constraints. The simplest choice is a local algorithm, where the plaquette variables on an embedded 3-cube are suitably changed by \pm 1 , and single plaquettes together with the matter flux at their boundary are changed by \pm 1 (see, e.g., [50, 51, 52, 59, 71]). However, for many values of the parameters such a local algorithm is not very efficient and better strategies are needed.

A powerful tool for the update of dual representations of spin systems or dual versions of lattice field theories with only matter fields is the Prokof'ev - Svistunov worm algorithm [99]. In these cases one has only loops of matter flux and the worm algorithm starts with violating the constraints at some site and then randomly propagates this defect through the lattice until the worm returns to the starting site and the violation of the constraint is healed. Each step is accepted with a Metropolis decision and the algorithm can be shown to be ergodic.

It is interesting to note that the idea of the worm algorithm can be generalized to abelian gauge Higgs systems. Again one violates the constraints at a randomly chosen position on the lattice and propagates the defect until the worm closes in a final step where the defect is healed. For systems with gauge and matter fields two types of constraints appear: Conservation of matter flux at each site of the lattice and conservation of the combined flux of matter and plaquette variables at each link. Thus the initial step violates both types of constraints and the individual steps of the generalized worm algorithm have to propagate the defects for both constraints simultaneously.

A suitably generalization of the worm algorithm to abelian gauge Higgs systems, the so called "surface worm algorithm" (SWA), was introduced in $[59,60,70]^{4}$. In an initial step the matter flux at a randomly chosen link is changed by \pm 1 , which violates the constraint at the particular link and at the two sites at the two endpoints of the link. Subsequently the defects are transported through the lattice with elements of surfaces ("segments") that consist of plaquettes where the corresponding $p$-variable and the matter flux at two of the sides of the plaquette is changed suitably by \pm 1 . In a final step the worm randomly adds an element such that all constraints are healed (see [59] for a detailed description). The acceptance of each step is governed by a Metropolis decision. In Fig. 2 we show an example for a short SWA on an initially empty lattice.

\section{Some results for the U(1) gauge Higgs system}

Let us now come to discussing examples of typical results that can be obtained with dual simulations. We use the U(1) gauge Higgs model with two flavors of opposite charge [70, 71]. In this section we present results for observables that can be obtained as derivatives of $\ln Z$ with respect to the parameters of the theory, i.e., masses, couplings and the chemical potential. These derivatives can be taken for both, the conventional representation, where the corresponding observables can be interpreted, as well as in the dual representation to obtain their form for the dual simulation. In the dual world these bulk observables typically assume the form of weighted sums over the dual variables. Two examples are the plaquette $\langle U\rangle$ and the particle number density $\langle n\rangle$ :

\footnotetext{
${ }^{4}$ For other attempts to generalize the worm concept to systems with gauge surfaces see $[53,54,55,56,57,58]$.
} 
1

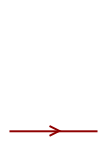

2

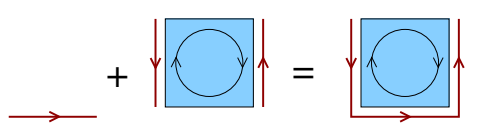

3

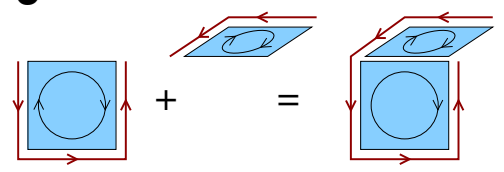

4

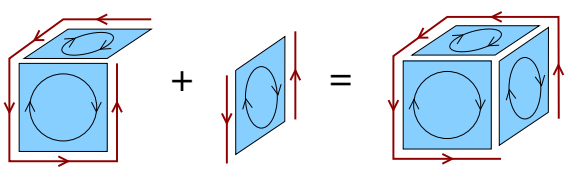

5

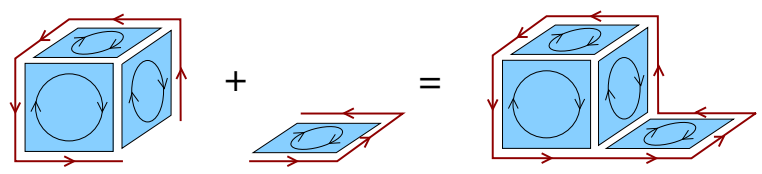

Figure 2: Example of updates with the surface worm algorithm (SWA): After randomly placing a link flux (step 1), the corresponding defect is propagated through the lattice by attaching plaquettes with two of their sides saturated with link flux (steps 2,3,4). The surface worm closes by inserting an element that heals the violated constraint (step 5). Each step is accepted with a Metropolis decision.

$$
\langle U\rangle=\frac{\partial \ln Z}{6 N_{s}^{3} N_{t} \partial \beta}=\frac{1}{6 N_{s}^{3} N_{t}}\left\langle\prod_{x, \rho<v} \frac{I_{p_{x, \rho v}}(\beta)^{\prime}}{I_{p_{x, \rho v}}(\beta)}\right\rangle,\langle n\rangle=\frac{\partial \ln Z}{6 N_{s}^{3} N_{t} \partial \mu}=\frac{1}{N_{s}^{3} N_{t}}\left\langle-\sum_{x} l_{x, 4}\right\rangle,
$$

where the prime in the first equation indicates the derivative with respect to $\beta$ and the expressions on the right hand sides are evaluated in the dual representation.

The observables (4.1) together with $\left\langle|\phi|^{2}\right\rangle=\partial / \partial M^{2} \ln Z / N_{s}^{3} N_{t}$, as well as the corresponding susceptibilities $\chi_{U}, \chi_{n}$ and $\chi_{|\phi|^{2}}$ were studied in $[70,71]$ for the $\mathrm{U}(1)$ gauge Higgs model with two flavors of opposite charge. The mass parameters and the chemical potentials of the two flavors were set to equal values $\left(M^{2}\right.$ and $\left.\mu\right)$.

For a first scan through parameter space, in Fig. 3 we show the observables $\langle U\rangle,\left\langle|\phi|^{2}\right\rangle$, and $\langle n\rangle$ (left to right) as a function of $\beta$ and $M^{2}$ for different values of the chemical potential, $\mu=$ $0.0,0.5,1.0$ and 1.5 (top to bottom). With the exception of $\langle n\rangle$ at $\mu=0$, where $\langle n\rangle \equiv 0$, in all observables we see the three phases expected for the U(1) gauge Higgs model: A confining phase characterized by $\langle U\rangle$ small, $\left\langle|\phi|^{2}\right\rangle \sim 0$ and $\langle n\rangle \sim 0$, a Coulomb phase $\left(\langle U\rangle \sim 0.6-0.8,\left\langle|\phi|^{2}\right\rangle \sim 0\right.$, $\langle n\rangle \sim 0)$, and a Higgs phase $\left(\langle U\rangle \sim 1.0,\left\langle|\phi|^{2}\right\rangle\right.$ and $\langle n\rangle \sim 0$ growing with $\left.-m^{2}\right)$.

The phases are separated by phase boundaries, which partly are signaled by very abrupt changes of the observables, in particular for the strong first order transition between the Higgsand the confinement phase. Increasing $\mu$ makes the transitions even more pronounced and in addition leads to a small shift of the phase boundaries in the $\beta-M^{2}$ plane.

For the case of $\mu=0$, the phase boundaries were determined in detail [70, 71] using the susceptibilities $\chi_{U}, \chi_{n}$ and $\chi_{|\phi|^{2}}$. The results are shown in Fig. 4. The boundary between the Higgsand the confinement phase is characterized by a strong first order transition, the boundary between Coulomb- and confining phase is of weak first order, and between the Higgs- and the Coulomb phase we found a continuous transition.

For two different combinations of the parameters $M^{2}$ and $\beta$, one in the Higgs phase, one in the confining phase (in Fig. 4 the two corresponding points are marked with red crosses) we now study 

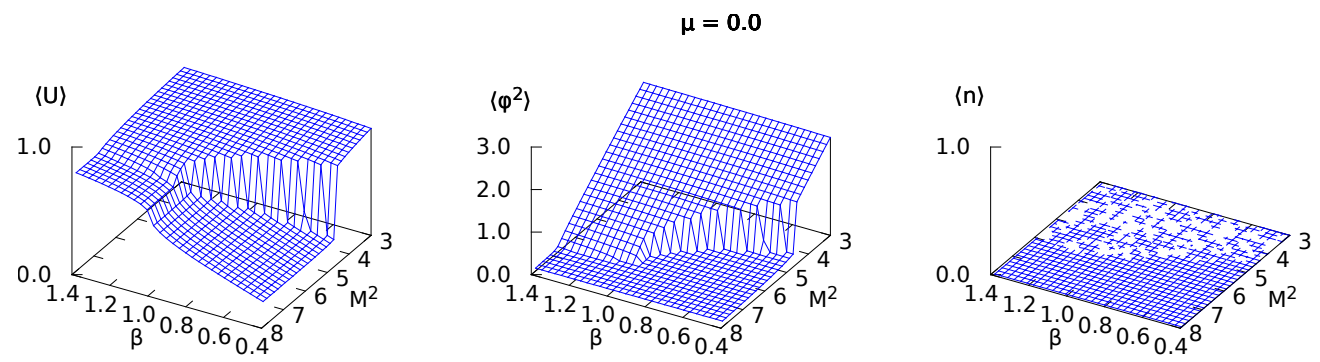

$$
\mu=0.5
$$
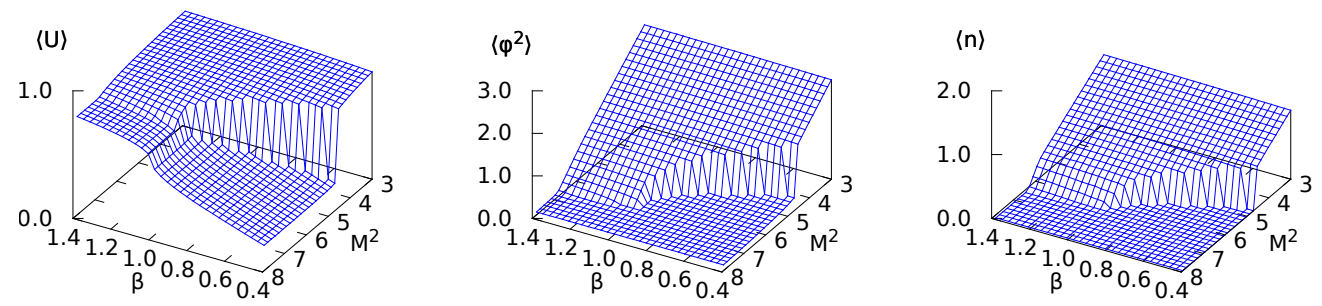

$$
\mu=1.0
$$
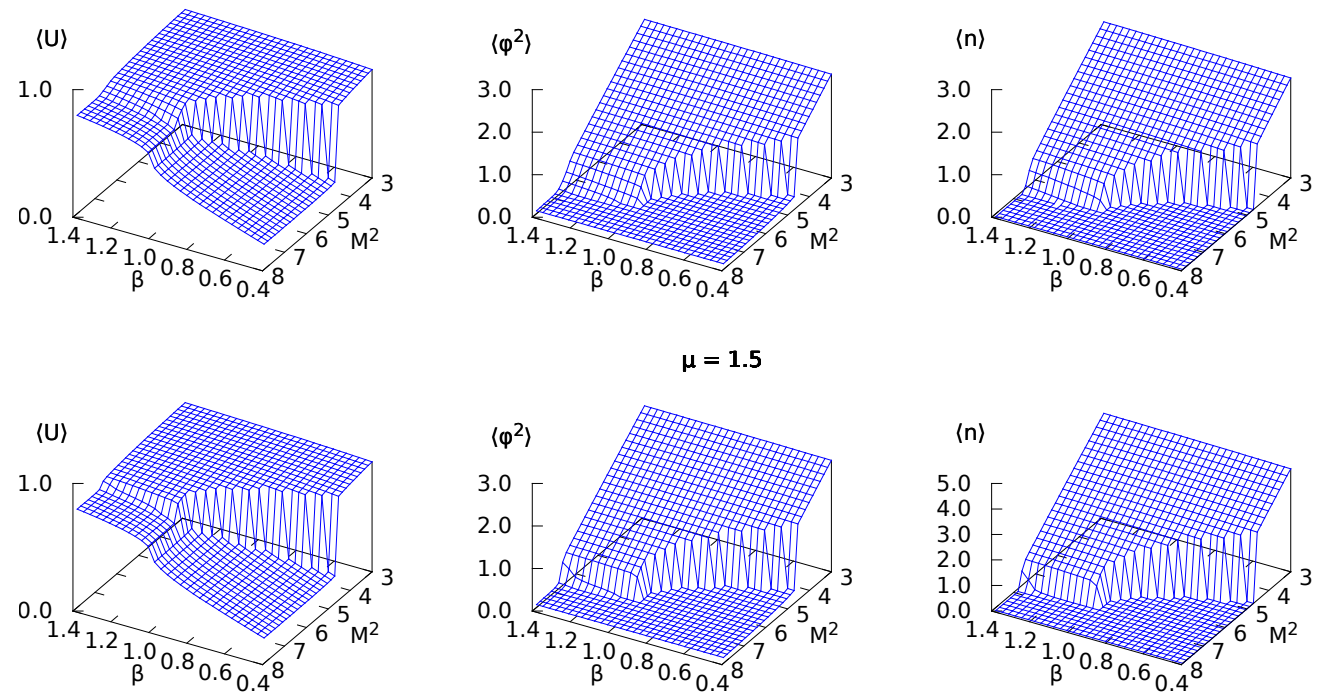

Figure 3: The observables $\langle U\rangle,\left\langle|\phi|^{2}\right\rangle$, and $\langle n\rangle$ as a function of $\beta$ and $M^{2}$ for different chemical potentials $\mu=0.0,0.5,1.0$ and 1.5. It can be seen how the phase boundaries shift with increasing chemical potential.

how observables depend on the chemical potential $\mu$. In Fig. 5 we show $\langle U\rangle,\left\langle|\phi|^{2}\right\rangle$ and $n$, as well as some of the dual occupation numbers versus $\mu$. The top row of plots is for the point in the Higgs phase $\left(\beta=0.75, M^{2}=5.73\right)$ and the bottom row for the confined phase $\left(\beta=0.75, M^{2}=4.92\right)$.

The general behavior in the Higgs phase (top row in Fig. 5) is the same for all observables: They are independent of the chemical potential $\mu$ up to $\mu=\mu_{c} \sim 2.65$. At this value there is a prominent first order jump followed by an essentially linear behavior for $\mu>\mu_{c}$. This finding is an example of the Silver Blaze behavior, i.e., the phenomenon that observables are independent of $\mu$ as long as the chemical potential is smaller than the lowest mass in the spectrum of excitations. In 


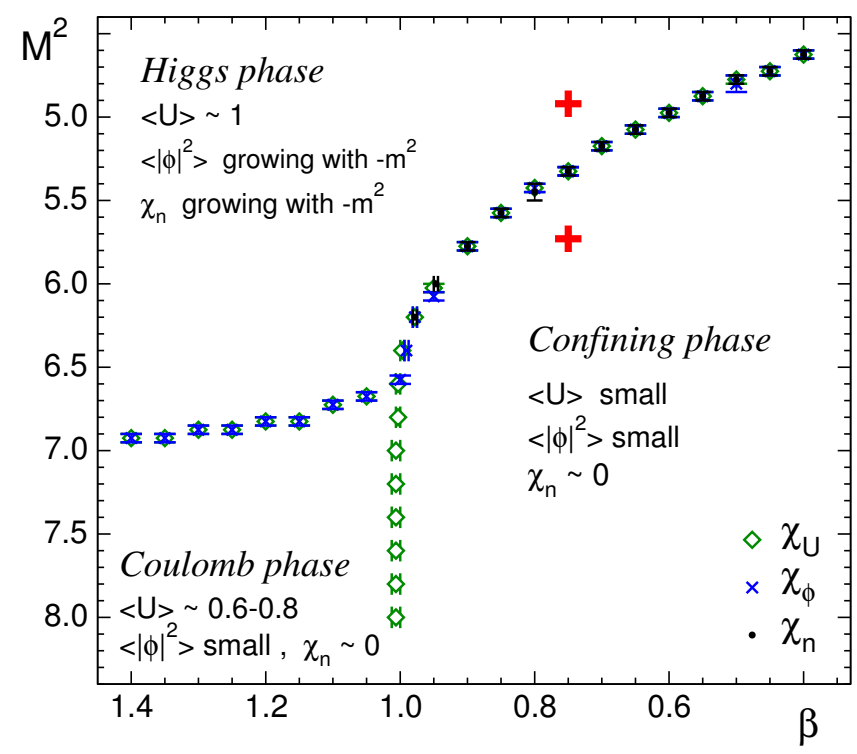

Figure 4: Phase diagram in the $\beta-M^{2}$ plane at $\mu=0$. We show the phase boundaries determined from the maxima of the susceptibilities $\chi_{U}$ and $\chi_{\phi}$ and the inflection points of $\chi_{n}$.
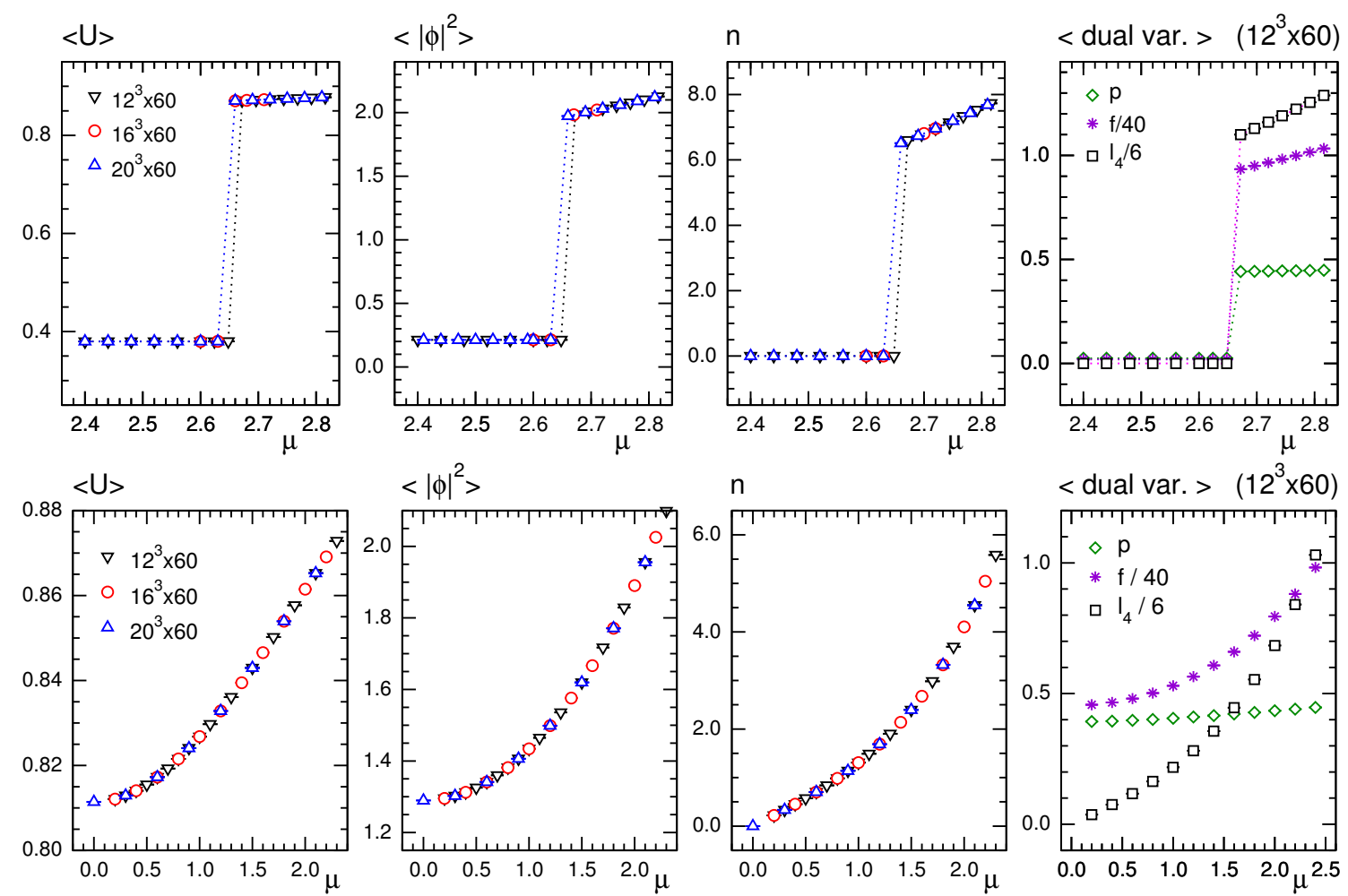

Figure 5: The observables $\langle U\rangle,\left\langle|\phi|^{2}\right\rangle, n$ (left to right) and the occupation numbers of dual variables as a function of $\mu$. We compare the results for parameters in the confined phase $\left(\beta=0.75, M^{2}=4.92\right.$, top row of plots) and in the Higgs phase ( $\beta=0.75, M^{2}=5.73$, bottom). 
[64] it was shown for the charged $\phi^{4}$ field that indeed $\mu_{c}$ very precisely coincides with the mass of the lowest excitation as determined in a spectroscopy calculation. The situation is different in the Coulomb phase (bottom row of plots in Fig. 5): There a non-trivial $\mu$-dependence sets in already at $\mu=0$. There is no mass gap, and thus no Silver Blaze type of behavior.

\section{Spectroscopy with dual variables}

Having discussed results for the bulk observables that can easily be expressed as moments of weighted sums of dual variables, let us now come to the question of how to implement $n$-point functions in a dual representation. The most general way is to add source terms $\sum_{x} \phi_{x} j_{x}$ to the action (2.2) of the matter fields. Again the dual transformation can be carried out in essentially the same way, with the main difference that one has additional dual variables that serve as sources and sinks for matter flux. Thus instead of closed loops, one has open strings as the dual degrees of freedom for the matter fields. The situation is somewhat simpler if one considers $n$-point functions. As an example we discuss the dual representation of 2-point functions for the charged $\phi^{4}$ field, i.e., the theory described by the action $S_{M}$ of Eq. (2.2) with $U_{x, v}=1$.

In the conventional representation the 2-point functions are given by

$$
\left\langle\phi_{y} \phi_{z}^{*}\right\rangle=\frac{1}{Z} \int D[\phi] e^{-S_{M}} \phi_{y} \phi_{z}^{*} \equiv \frac{1}{Z} Z_{y, z},
$$

where $Z_{y, z}$ is used to denote the partition sum with two field insertions $\phi_{y}$ and $\phi_{z}^{*}$. The derivation of Section 2 is easily generalized to find the dual representation of $Z_{y, z}$,

$$
\begin{aligned}
Z_{y, z}= & \sum_{\{\bar{l}, l\}} e^{-\mu \sum_{x} l_{x, 4}} \prod_{x} \delta\left(\sum_{v}\left[l_{x, v}-l_{x-\widehat{v}, v}\right]-\delta_{x, y}+\delta_{x, z}\right) \\
& \times \prod_{x, v} \frac{1}{\left(\left|l_{x, v}\right|+\bar{l}_{x, v}\right) ! \bar{l}_{x, v} !} P\left(\sum_{v}\left[\left|l_{x, v}\right|+\left|l_{x-\widehat{v}, v}\right|+2\left(\bar{l}_{x, v}+\bar{l}_{x-\widehat{v}, v}\right)\right]+\delta_{x, y}+\delta_{x, z}\right) .
\end{aligned}
$$

At the positions $y$ and $z$ where the source fields $\phi_{y}$ and $\phi_{z}^{*}$ are located the index of the weight factors $P(n)$ is increased (see the Kronecker deltas in the argument of the $P$ ), and also the constraints for the $l$-fluxes have additional terms at the sites $y$ and $z$, such that a flux can start at $y$ and end at $z$. Thus in the dual representation for $Z_{y, z}$ the set of configurations of allowed $l$-flux consists of closed loops and a single open line of flux connecting the sites $y$ and $z$.

For an efficient evaluation of $Z_{y, z}$ it was proposed [61] to consider a generalized partition function $\mathscr{Z}$ where one sums over all possible positions of the field insertions, $\mathscr{Z} \equiv \sum_{u, v} Z_{u, v}$. The configurations that constitute $\mathscr{Z}$ consist of closed loops of flux plus a single flux line with open ends at arbitrary sites $u$ and $v$ of the lattice. For the Monte Carlo simulation of $\mathscr{Z}$ we may reuse the standard worm algorithm, and view every step of the worm as an admissible configuration contribution to $\mathscr{Z}$ (not only the configurations where the worm has closed that contribute to $Z$ ). The two point functions are then obtained as

$$
\left\langle\phi_{y} \phi_{z}^{*}\right\rangle=\frac{Z_{y, z}}{Z}=\frac{\left\langle\delta_{u, y} \delta_{v, z}\right\rangle_{\mathscr{Z}}}{\left\langle\delta_{u, v} P\left(f_{u}\right) / P\left(f_{u}+2\right)\right\rangle_{\mathscr{Z}}},
$$

where $\langle. .\rangle_{\mathscr{Z}}$ denotes the expectation value with respect to the enlarged ensemble $\mathscr{Z}$. Here $f_{u}=$ $\sum_{v}\left[\left|l_{u, v}\right|+\left|l_{u-\widehat{v}, v}\right|+2\left(\bar{l}_{u, v}+\bar{l}_{u-\widehat{v}, v}\right)\right]$ denotes the combined $l$ - and $\bar{l}$-flux at site $u$ that enters the 


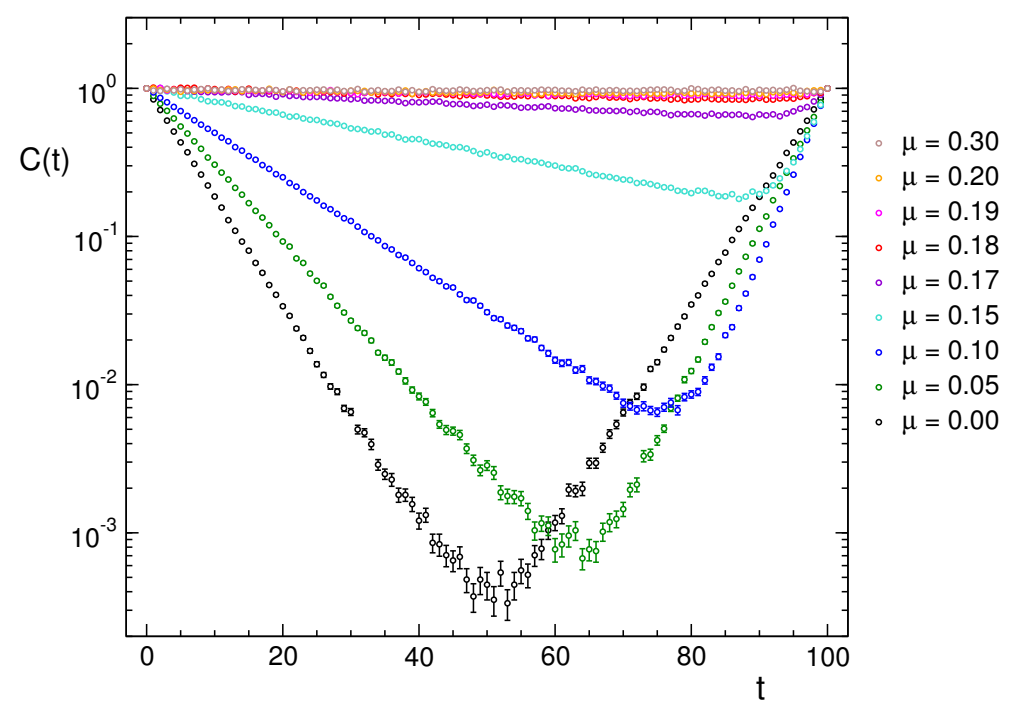

Figure 6: Zero momentum propagator as a function of euclidean time $t$ for the charged $\phi^{4}$ field at zero and non-zero chemical potential.

weights $P$, and the reweighting with $P\left(f_{u}\right) / P\left(f_{u}+2\right)$ in (5.3) is necessary to correctly obtain the original partition sum $Z$ in the denominator of (5.3).

Based on these techniques, in $[62,63]$ the 2-point functions for the charged $\phi^{4}$ field were evaluated for finite $\mu$. In Fig. 6 we show the results for the 2-point function projected to zero momentum as a function of the euclidean time separation $t$. The results for different values of $\mu$ are compared. While for $\mu=0$ the propagator has the conventional form of symmetrical forward and backward propagation in time, this is different for non-zero chemical potential. As is obvious from Fig. 6, for $\mu>0$ one finds a different slope for forward and backward propagation. The asymmetry increases with increasing $\mu$ until $\mu$ becomes so large that Bose-Einstein condensation sets in (here at $\mu \sim 0.17$ ), and the propagator becomes flat. The propagators were analyzed quantitatively and it was found that the exponents that describe the decay for forward and backward propagation are given by $m \pm \mu$, where $m$ is the renormalized mass extracted from the $\mu=0$ propagator. The results from this simple example show that there is no principal obstacle for dual spectroscopy at finite density - at least for the simple theory studied here.

\section{Dualized models as reference systems}

As we have already outlined in the introduction an important application of finite density systems where a simulation with dual methods is possible is their use as reference systems for testing and assessing other approaches to finite density such as series expansions, stochastic methods et cetera. In this section we report about two such comparisons.

An interesting new development for stochastic techniques is the formulation and simulation of quantum field theories on a Lefschetz thimble [27]-[31], a manifold that generalizes the notion of curves of steepest descent to higher dimensions. As for the complex Langevin approach the degrees of freedom are generalized to complex numbers and the Lefschetz thimble is then defined as the 


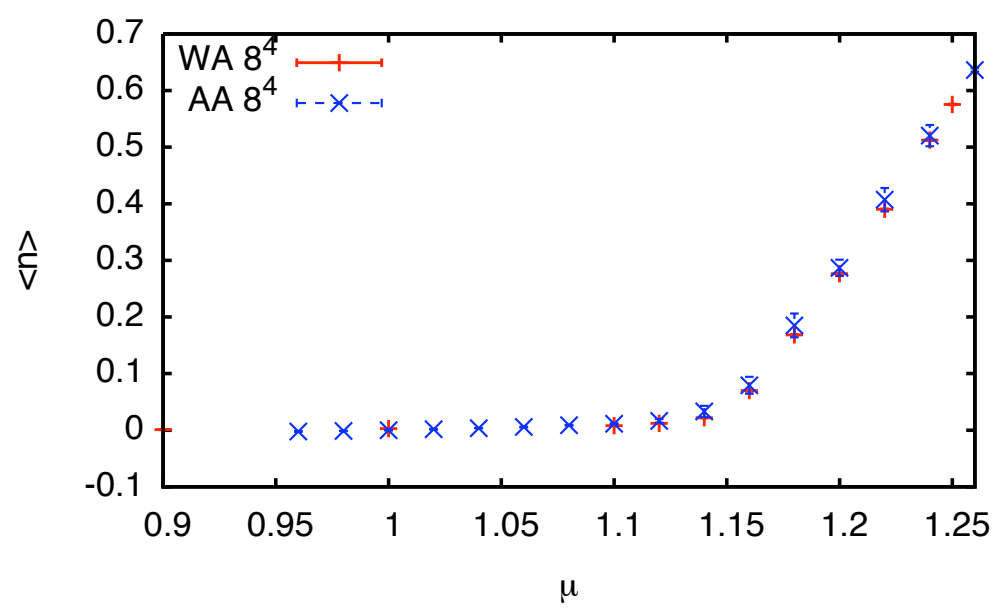

Figure 7: Comparison of results from the Lefschetz thimble approach (labelled AA) to the outcome of a dual simulation (WA). The plot from [29] shows the particle number as function of $\mu$ for the charged $\phi^{4}$-field.

path in the complex plane where the imaginary part of the action is constant and the corresponding constant phase of the Boltzmann factor cancels.

The development of the method has reached a stage where simulations of various lattice models were presented, one of them being the charged $\phi^{4}$ model at finite chemical potential [29], i.e., the theory described by the action $S_{M}$ of Eq. (2.2) with $U_{x, v}=1$. For this system one can compare to the results from the dual simulation [64] and in this way assess the newly developed method.

In Fig. 7 we compare the results for the particle number density $\langle n\rangle$ as a function of $\mu$. The curve shows the onset of condensation at $\mu \sim 1.14$ (the transition is somewhat rounded since the simulation is for a $8^{4}$ lattice where finite temperature effects are non-negligible). It is obvious from the plot, that the results from the Lefschetz thimble approach and from the dual simulation agree quite well. Another example of a comparison of stochastic methods to a dual simulation is the test of complex Langevin techniques in the SU(3) spin model [19, 65, 66, 67].

A further interesting application of systems accessible to dual techniques is their use as reference models for various expansions in the chemical potential $\mu$. The idea is to expand around the $\mu=0$ theory, where the complex action problem is absent and thus the expansion coefficients can be computed with conventional simulations. The simplest such expansion is the Taylor series, i.e., the partition function $Z$ (or also the free energy) is expanded in $\mu^{2}$ ( $Z$ is an even function in $\mu$ ). However, it is not a-priori clear whether this choice of functions to expand in is optimal. An alternative $[72,73]$ from statistical mechanics is the fugacity expansion where $Z$ is expanded in a Laurent series in the fugacity variable $z=e^{\mu \beta}$.

To assess the two expansions, fugacity and Taylor series, they were evaluated in the $\mathbb{Z}_{3}$ spin model, where a dual simulation is available for reference [35]-[38]. The system is a simple effective theory for the Polyakov loop with an action given by

$$
S=-\sum_{x}\left[\tau \sum_{v=1}^{3}\left[P_{x} P_{x+\hat{v}}^{*}+c . c .\right]+\kappa e^{\mu} P_{x}+\kappa e^{-\mu} P_{x}^{*}\right],
$$

where the sum runs over the sites $x$ of a 3-d lattice and the Polyakov loop variables $P_{x}$ are elements 

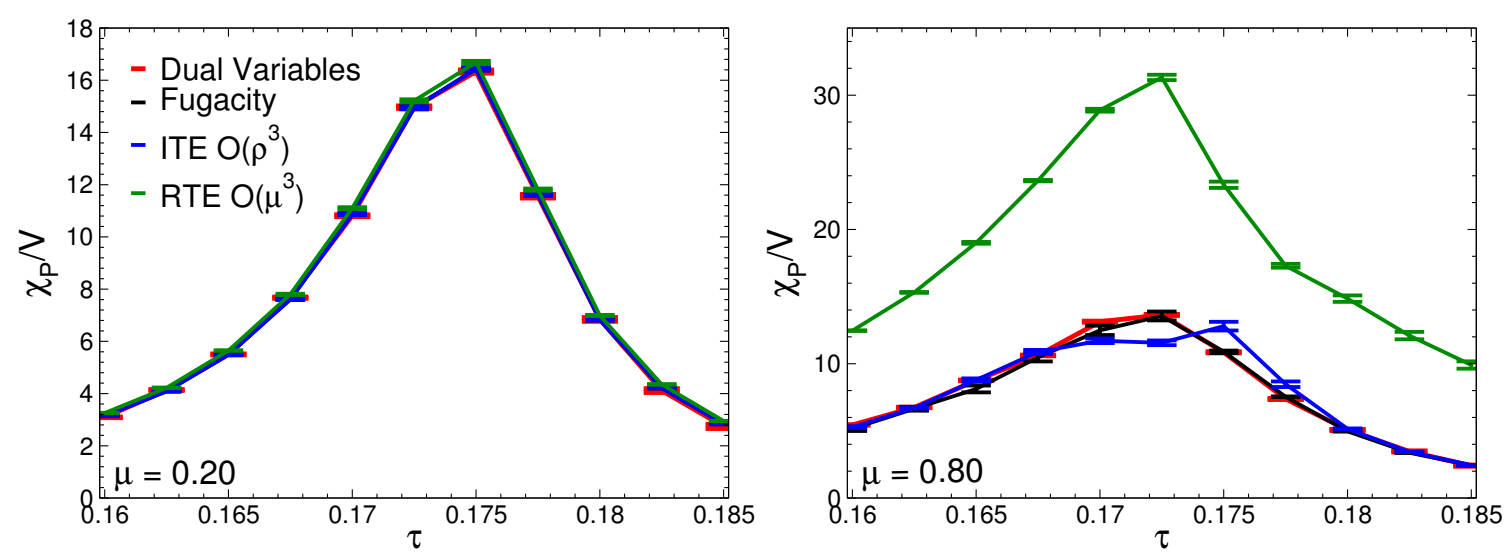

Figure 8: Comparison of various series expansions in $\mu$ for susceptibility $\chi_{P} / V$ in the $\mathbb{Z}_{3}$ spin model to the results from a dual simulation. We show $\chi_{P}$ from fugacity expansion, improved Taylor expansion (ITE) and regular Taylor expansion (RTE) as a function of the temperature parameter $\tau$ for two different values of the chemical potential, $\mu=0.2$ (lhs. plot) and $\mu=0.8$ (rhs.).

of $\mathbb{Z}_{3}=\left\{1, e^{ \pm 2 i \pi / 3}\right\} . \tau$ is a increasing function of the temperature, $\kappa$ a function that decreases with increasing quark mass and $\mu$ is the chemical potential. For $\mu \neq 0$ the action is complex.

The Taylor expansion, the fugacity expansion and a modified Taylor expansion (power series in $e^{ \pm \mu}-1$ ) are worked out easily and can be compared to the results of the dual approach. Fig. 8 shows the results for the Polyakov loop susceptibility as a function of the temperature parameter $\tau$ at $\kappa=0.01$ for two different values of the chemical potential, $\mu=0.2$ in the lhs. plot and $\mu=0.8$ on the rhs. The figures show that at $\mu=0.2$ all three series expansions (Taylor and improved Taylor expansion are up to third order) agree quite well with the dual simulation results, while for $\mu=0.8$ the regular Taylor expansion is far off the dual curve, the improved Taylor series starts to have problems, and only the fugacity expansion still reasonably matches the dual results.

In another project the recent developments for the density of states approach $[116,117,118]$ will be implemented in the system (6.1) and cross-checked with the dual results. We expect that model studies in systems where a dual simulation is possible can help to optimize new ideas and strategies for finite density before applying them in considerably more expensive realistic theories.

\section{Fermions}

In this section we briefly discuss aspects of dual representations for systems with fermionic matter. As a toy example we consider a system of staggered fermions with a simple quartic interaction. The action is given by

$$
S=\sum_{x}\left(m \bar{\psi}_{x} \psi_{x}+\sum_{v} \frac{\eta_{v}(x)}{2}\left[e^{\mu \delta_{v 4}} \bar{\psi}_{x} \psi_{x+\hat{v}}-e^{-\mu \delta_{v 4}} \bar{\psi}_{x} \psi_{x-\hat{v}}\right]-g \sum_{\langle x y\rangle} \bar{\psi}_{x} \psi_{x} \bar{\psi}_{y} \psi_{y}\right) .
$$

Here $\bar{\psi}_{x}, \psi_{x}$ are single component Grassmann valued fields at the sites $x$ of the lattice, $\eta_{v}(x)=$ $(-1)^{\sum_{\alpha=1}^{\nu-1} x_{\alpha}}$ is the staggered sign factor which plays the role of the Dirac matrices, $m$ the fermion mass, $g$ the quartic coupling and the last sum runs over all neighbors $y$ of the site $x$. 
Similar to the case of the bosonic model considered in Section 2, the Boltzmann factor $e^{-S}$ in the path integral can be factorized into a product of individual terms that live on links and on sites. Each of these factors can be expanded, and since Grassmann variables are nilpotent, the expansion terminates after the second term, such that we get for the example of a mass term at site $x$ the series $e^{-m \bar{\psi}_{x} \psi_{x}}=\sum_{s_{x}=0}^{1}(-m)^{s_{x}}\left(\bar{\psi}_{x} \psi_{x}\right)^{s_{x}}$ and similar for the other terms. The expansion indices $\left(s_{x}\right.$ in our example) can have only two values, $s_{x} \in\{0,1\}$, and thus can be interpreted as "activation indices" that activate $\left(s_{x}=1\right)$ certain terms or turn them off $\left(s_{x}=0\right)$. For a non-vanishing result of the Grassmann integral $Z=\int \prod_{x} d \bar{\psi}_{x} d \psi_{x} e^{-S}$ each $\psi_{x}$ and each $\bar{\psi}_{x}$ must be activated exactly once. This leads to constraints for the allowed configurations of the activation indices, which again give rise to loops of dual variables living on links from the hopping terms, but also insertions of "monomers" from the mass and quartic coupling terms which completely saturate the Grassmann integrals at all sites that they are activated on.

A serious complication comes from the fact that for the evaluation of the Grassmann integral the individual variables $\bar{\psi}_{x}$ and $\psi_{x}$ have to be brought into a canonical order, which due to the anticommutating nature of the Grassmann variables gives rise to additional minus signs. For example every loop of dual flux comes with a minus sign. Further minus signs that depend on the shape of the loops come from the staggered factors $\eta_{v}(x)$. Thus although we do have a dual representation of the system (where again the chemical potential enters in a harmless way), the complex action problem is not solved, since the configurations of the dual variables come with both signs. Although examples are known (in particular in low dimensions) where this simple dualization leads to a form useful for simulations [81]-[90], the general perspective is not very good for this approach.

An interesting concept is the so-called fermion bag approach [91]-[97]: There only the Boltzmann factor of the interaction term is expanded. If the interaction is such that the corresponding activated terms completely saturate the Grassmann integral locally (this is the case for our example), the sites where the interaction is activated can be removed from the Grassmann integral. What remains is a free theory (only hopping and mass terms) on a lattice where sites are removed. The partition function turns into an unconstrained sum over all configurations of the activation indices for the fermion interaction, and the method becomes efficient in a parameter range where many interaction terms are activated and the terms of the free theory need to be evaluated only on small patches of the lattice (fermion bags). Several interesting models could be studied with the fermion bag approach and we refer the reader to the lecture series [98] by Chandrasekharan for an overview and the discussion of further aspects of the fermion bag method and related ideas.

\section{Strong coupling and large mass}

In the first few sections of this contribution we have shown that for bosonic matter with abelian symmetries the problem of solving the complex action problem by mapping the system to dual variables is essentially solved. In the previous section it was discussed that for fermions the challenge is much harder due to the extra minus signs of the matter loops from the anti-commutation relations of the fermions and signs from the Dirac matrices. Similarly difficult is the question for a suitable dual representation of non-abelian gauge fields (for some attempts see [100]-[103]).

It is, however, well known that in the limits of large quark mass $m$ and strong coupling one can compute the leading terms of a dual representation, and that these leading terms are better 


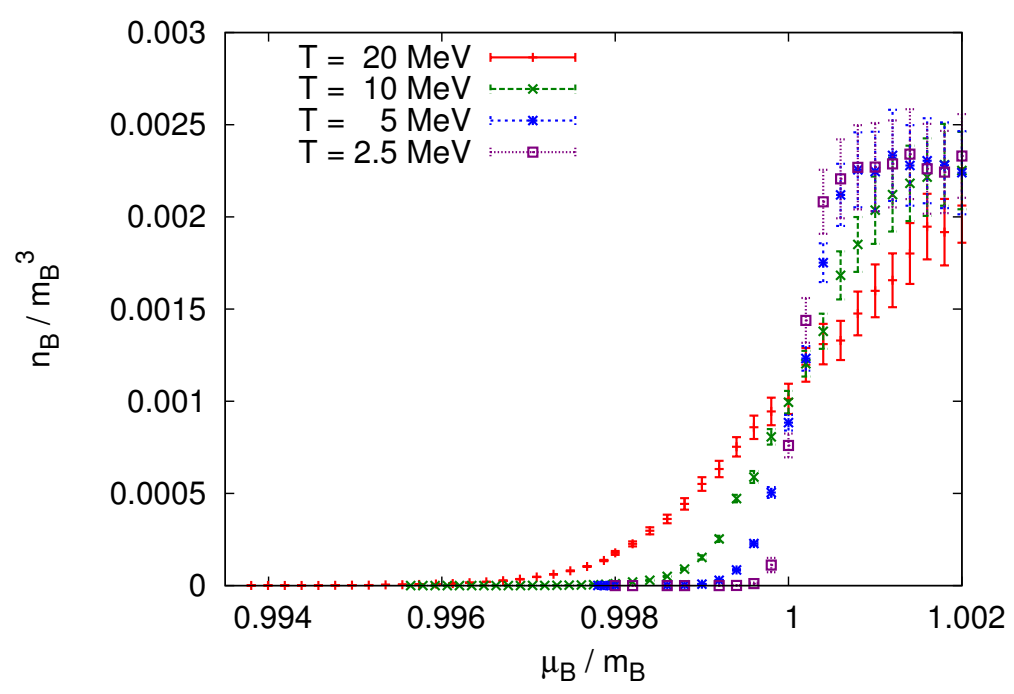

Figure 9: Particle number density as a function of the chemical potential for lattice QCD in the strongcoupling / large-mass limit. (Figure from [106].)

behaved from a Monte Carlo point of view: Large mass $m$ suppresses long matter loops, as each link of matter flux costs a weight factor $\propto 1 / \mathrm{m}$. In a similar way each occupied plaquette of a gauge surface costs a factor $\beta$, and the inverse gauge coupling $\beta$ is small in the strong coupling limit. Several attempts to study such partially dualized theories in the large-mass/strong-coupling limits can be found in the literature (see, e.g., [74]-[80], [92], [104]-[108]).

A particularly elaborate implementation of a dualization in the large-mass/strong-coupling limit has been presented in [104]-[108]. The leading terms of the dual representation in the largemass and strong-coupling limits were taken into account, but the coefficients were determined with a matching procedure to optimally reproduce physics at $\mu=0$. The resulting dual theory was then studied at finite $\mu$, and as an example of what may be achieved, Fig. 9 shows the baryon number density as a function of the chemical potential for different temperatures. One nicely sees the condensation transition becoming sharper as the temperature is decreased.

It is clear that dual simulations in the large-mass/strong-coupling region have considerable limitations - nevertheless the results that were obtained recently nicely illustrate physical mechanism that play an important role also at physical parameter values.

\section{Concluding remarks}

In this contribution we have discussed recent developments for attempts to overcome the complex action problem of lattice field theories by the use of transformations to new variables ("dual variables") where the partition sum has only real and positive contributions. Our main example was the U(1) gauge Higgs model which is characteristic for all abelian gauge Higgs models, a class of systems where the finite density complex action problem is essentially solved. Dual representations have the form of conserved discretized fluxes for matter fields and surfaces that are either closed or bounded by matter flux for the gauge degrees of freedom. We discussed new algorithmic ideas for 
dual simulations and typical examples for results that were obtained, including results for 2-point functions and spectroscopy.

For fermion systems and non-abelian symmetries the progress is not as far advanced, although the general structure of loops and surfaces is the same. For fermions the Grassmann variables and the Dirac matrices give rise to additional minus signs for the matter flux and the non-abelian nature of the gauge fields prevents one from the reordering of the link variables that led to a successful dualization in the abelian case. We stress, however, that the abelian and bosonic examples show that dual representations are not unique and thus there is hope that further interesting ideas and developments might also be possible for fermions and systems with non-abelian symmetries.

\section{References}

[1] G. Aarts, PoS LATTICE 2012 (2012) 017 [arXiv:1302.3028 [hep-lat]].

[2] P. Petreczky, PoS ConfinementX (2012) 028 [arXiv:1301.6188 [hep-lat]].

[3] L. Levkova, PoS LATTICE 2011 (2011) 011 [arXiv:1201.1516 [hep-lat]].

[4] S. Gupta, PoS LATTICE 2010 (2010) 007 [arXiv:1101.0109 [hep-lat]].

[5] U. Wolff, PoS LATTICE 2010 (2010) 020 [arXiv:1009.0657 [hep-lat]].

[6] P. de Forcrand, PoS LAT 2009 (2009) 010 [arXiv:1005.0539 [hep-lat]].

[7] A. Li, PoS LAT 2009 (2009) 011 [arXiv:1002.4459 [hep-lat]].

[8] S. Chandrasekharan, PoS LATTICE 2008 (2008) 003 [arXiv:0810.2419 [hep-lat]].

[9] S. Ejiri, PoS LATTICE 2008 (2008) 002 [arXiv:0812.1534 [hep-lat]].

[10] G. Aarts, arXiv:1312.0968 [hep-lat].

[11] L. Bongiovanni, G. Aarts, E. Seiler, D. Sexty and I.-O. Stamatescu, arXiv:1311.1056 [hep-lat].

[12] G. Aarts, L. Bongiovanni, E. Seiler, D. Sexty and I.-O. Stamatescu, arXiv:1310.7412 [hep-lat].

[13] D. Sexty, arXiv:1310.6186 [hep-lat].

[14] P. Giudice, G. Aarts and E. Seiler, arXiv:1309.3191 [hep-lat].

[15] D. Sexty, arXiv:1307.7748 [hep-lat].

[16] G. Aarts, P. Giudice and E. Seiler, Annals Phys. 337 (2013) 238 [arXiv:1306.3075 [hep-lat]].

[17] G. Aarts, L. Bongiovanni, E. Seiler, D. Sexty and I.-O. Stamatescu, Eur. Phys. J. A 49 (2013) 89 [arXiv:1303.6425 [hep-lat]].

[18] G. Aarts, F.A. James, J.M. Pawlowski, E. Seiler, D. Sexty, I.-O. Stamatescu, JHEP 1303 (2013) 073 [arXiv:1212.5231 [hep-lat]].

[19] G. Aarts and F.A. James, JHEP 1201 (2012) 118 [arXiv:1112.4655 [hep-lat]].

[20] G. Aarts, F.A. James, E. Seiler, I.-O. Stamatescu, Eur. Phys. J. C 71 (2011) 1756 [arXiv:1101.3270].

[21] G. Aarts and K. Splittorff, JHEP 1008 (2010) 017 [arXiv:1006.0332 [hep-lat]].

[22] G. Aarts and F.A. James, JHEP 1008 (2010) 020 [arXiv:1005.3468 [hep-lat]].

[23] G. Aarts, E. Seiler and I.-O. Stamatescu, Phys. Rev. D 81 (2010) 054508 [arXiv:0912.3360 [hep-lat]]. 
[24] G. Aarts, F.A. James, E. Seiler, I.-O. Stamatescu, Phys. Lett. B 687 (2010) 154 [arXiv:0912.0617].

[25] G. Aarts, Phys. Rev. Lett. 102 (2009) 131601 [arXiv:0810.2089 [hep-lat]].

[26] G. Aarts, JHEP 0905 (2009) 052 [arXiv:0902.4686 [hep-lat]].

[27] M. Cristoforetti, F. Di Renzo, A. Mukherjee and L. Scorzato, arXiv:1312.1052 [hep-lat].

[28] A. Mukherjee, M. Cristoforetti and L. Scorzato, Phys. Rev. D 88 (2013) 051502 [arXiv:1308.0233].

[29] M. Cristoforetti, F. Di Renzo, A. Mukherjee and L. Scorzato, Phys. Rev. D 88 (2013) 051501 [arXiv:1303.7204 [hep-lat]].

[30] M. Cristoforetti et al., Phys. Rev. D 86 (2012) 074506 [arXiv:1205.3996 [hep-lat]].

[31] G. Aarts, Phys. Rev. D 88 (2013) 094501 [arXiv:1308.4811 [hep-lat]].

[32] A. Patel, Nucl. Phys. B 243 (1984) 411; Phys. Lett. B 139 (1984) 394.

[33] T. DeGrand and C. DeTar, Nucl. Phys. B 225 (1983) 590.

[34] J. Condella and C. DeTar, Phys. Rev. D 61 (2000) 074023 [arXiv:hep-lat/9910028].

[35] Y. D. Mercado, H.G. Evertz, C. Gattringer, Phys. Rev. Lett. 106 (2011) 222001 [arXiv:1102.3096].

[36] Y.D. Mercado, H.G. Evertz and C. Gattringer, Comput. Phys. Commun. 183 (2012) 1920 [arXiv:1202.4293[hep-lat]].

[37] Y. Delgado, H.G. Evertz, C. Gattringer and D. Göschl, PoS LATTICE 2011 (2011) 190 [arXiv:1111.0916 [hep-lat]].

[38] Y. Delgado, H.G. Evertz, C. Gattringer, Acta Phys. Polon. Supp. B 4 (2011) 703 [arXiv:1110.6862].

[39] M. Hogervorst, U. Wolff, Nucl. Phys. B 855 (2012) 885 [arXiv:1109.6186 [hep-lat]].

[40] P. Weisz, U. Wolff, Nucl. Phys. B 846 (2011) 316 [arXiv:1012.0404 [hep-lat]].

[41] U. Wolff, Phys. Rev. D 79 (2009) 105002 [arXiv:0902.3100 [hep-lat]].

[42] U. Wolff, Nucl. Phys. B 832 (2010) 520 [arXiv:1001.2231 [hep-lat]].

[43] U. Wolff, Nucl. Phys. B 824 (2010) 254 [Erratum-ibid. 834 (2010) 395] [arXiv:0908.0284 [hep-lat]].

[44] U. Wolff, Phys. Rev. D 79 (2009) 105002 [arXiv:0902.3100 [hep-lat]].

[45] U. Wolff, Nucl. Phys. B 814 (2009) 549 [arXiv:0812.0677 [hep-lat]].

[46] U. Wolff, Nucl. Phys. B 810 (2009) 491 [arXiv:0808.3934 [hep-lat]].

[47] E. Grünwald, Y. D. Mercado and C. Gattringer, arXiv:1310.6520 [hep-lat].

[48] M. Wilfling and C. Gattringer, arXiv:1311.7436 [hep-lat].

[49] C. Gattringer and A. Schmidt, Phys. Rev. D 86 (2012) 094506 [arXiv:1208.6472 [hep-lat]].

[50] T. Sterling, J. Greensite, Nucl. Phys. B 220 (1983) 327.

[51] M. Panero, JHEP 0505 (2005) 066 [hep-lat/0503024].

[52] V. Azcoiti, E. Follana, A. Vaquero, G. Di Carlo, JHEP 0908 (2009) 008 [arXiv:0905.0639 [hep-lat]].

[53] M.G. Endres, Phys. Rev. D 75 (2007) 065012 [hep-lat/0610029].

[54] M.G. Endres, PoS LAT06 (2006) 133 [hep-lat/0609037].

[55] T. Korzec and U. Wolff, Nucl. Phys. B 871 (2013) 145 [arXiv:1212.2875 [hep-lat]]. 
[56] T. Korzec and U. Wolff, arXiv:1309.1331 [hep-lat].

[57] T. Korzec and U. Wolff, arXiv:1311.5198 [hep-lat].

[58] T. Korzec, U. Wolff, PoS LATTICE 2010 (2010) 029 [arXiv:1011.1359 [hep-lat]].

[59] Y. D. Mercado, C. Gattringer, A. Schmidt, Comput. Phys. Commun. 184 (2013) 1535 [arXiv:1211.3436 [hep-lat]].

[60] A. Schmidt, Y. D. Mercado, C. Gattringer, PoS LATTICE 2012 (2012) 098 [arXiv:1211.1573].

[61] T. Korzec, I. Vierhaus, U. Wolff, Comp. Phys. Comm. 182 (2011) 1477 [arXiv:1101.3452 [hep-lat]].

[62] C. Gattringer and T. Kloiber, Phys. Lett. B 720 (2013) 210 [arXiv:1212.3770 [hep-lat]].

[63] T. Kloiber and C. Gattringer, arXiv:1310.8535 [hep-lat].

[64] C. Gattringer and T. Kloiber, Nucl. Phys. B 869 (2013) 56 [arXiv:1206.2954 [hep-lat]].

[65] Y. D. Mercado and C. Gattringer, Nucl. Phys. B 862 (2012) 737 [arXiv:1204.6074 [hep-lat]].

[66] Y. Delgado and C. Gattringer, Acta Phys. Polon. Supp. 5, 1033 (2012) [arXiv:1208.1169 [hep-lat]].

[67] C. Gattringer, Nucl. Phys. B 850 (2011) 242 [arXiv:1104.2503 [hep-lat]].

[68] P.N. Meisinger and M.C. Ogilvie, arXiv:1306.1495 [hep-lat].

[69] P.N. Meisinger and M.C. Ogilvie, arXiv:1311.5515 [hep-lat].

[70] Y. D. Mercado, C. Gattringer, A. Schmidt, Phys. Rev. Lett. 111 (2013) 141601 [arXiv:1307.6120].

[71] Y. Delgado, C. Gattringer and A. Schmidt, arXiv:1311.1966 [hep-lat].

[72] J. Danzer and C. Gattringer, Phys. Rev. D 86 (2012) 014502 [arXiv:1204.1020 [hep-lat]].

[73] E. Bilgici, J. Danzer, C. Gattringer, C.B. Lang, L. Liptak, Phys. Lett. B 697 (2011) 85 [arXiv:0906.1088 [hep-lat]].

[74] P. Rossi and U. Wolff, Nucl. Phys. B 248 (1984) 105.

[75] F. Karsch and K.H. Mütter, Nucl. Phys. B 313 (1989) 541.

[76] S. Chandrasekharan, Int. J. Mod. Phys. B 20 (2006) 2714.

[77] W. Unger and P. de Forcrand, J. Phys. G 38 (2011) 124190 [arXiv:1107.1553 [hep-lat]].

[78] P. de Forcrand and M. Fromm, Phys. Rev. Lett. 104 (2010) 112005 [arXiv:0907.1915 [hep-lat]].

[79] P. de Forcrand and S. Kim, Phys. Lett. B 645 (2007) 339 [hep-lat/0608012].

[80] M. Fromm, Nucl. Phys. A 820 (2009) 179C.

[81] C. Gattringer, Nucl. Phys. B 543 (1999) 533 [hep-lat/9811014].

[82] C. Gattringer, Nucl. Phys. B 559 (1999) 539 [hep-lat/9903021].

[83] C. Gattringer, V. Hermann, M. Limmer, Phys. Rev. D 76 (2007) 014503 [arXiv:0704.2277 [hep-lat]].

[84] U. Wenger, Phys. Rev. D 80 (2009) 071503 [arXiv:0812.3565 [hep-lat]].

[85] V. Maillart and U. Wenger, PoS LATTICE 2010 (2010) 257 [arXiv:1104.0569 [hep-lat]].

[86] K. Steinhauer and U. Wenger, arXiv:1311.5403 [hep-lat].

[87] M.G. Endres, Phys. Rev. A 85 (2012) 063624 [arXiv:1204.6182 [hep-lat]]. 
[88] M.G. Endres, Phys. Rev. A 87 (2013) 063617 [arXiv:1302.6694 [hep-lat]].

[89] U. Wolff, Nucl. Phys. B 789 (2008) 258 [arXiv:0707.2872 [hep-lat]].

[90] O. Bär, W. Rath and U. Wolff, Nucl. Phys. B 822 (2009) 408 [arXiv:0905.4417 [hep-lat]].

[91] S. Chandrasekharan, Phys. Rev. D 82 (2010) 025007 [arXiv:0910.5736 [hep-lat]].

[92] S. Chandrasekharan and A. Li, JHEP 1101 (2011) 018 [arXiv:1008.5146 [hep-lat]].

[93] S. Chandrasekharan and A. Li, Phys. Rev. Lett. 108 (2012) 140404 [arXiv:1111.7204 [hep-lat]].

[94] S. Chandrasekharan and A. Li, Phys. Rev. D 85 (2012) 091502 [arXiv:1202.6572 [hep-lat]].

[95] S. Chandrasekharan, Phys. Rev. D 86 (2012) 021701 [arXiv:1205.0084 [hep-lat]].

[96] S. Chandrasekharan and A. Li, Phys. Rev. D 88 (2013) 021701 [arXiv:1304.7761 [hep-lat]].

[97] S. Chandrasekharan, Eur. Phys. J. A 49 (2013) 90 [arXiv:1304.4900 [hep-lat]].

[98] S. Chandrasekharan, Lectures at the Schladming Winter School for Theoretical Physics 2013, http://physik.uni-graz.at/schladming2013/index.php?sf=18

[99] N. Prokof'ev and B. Svistunov, Phys. Rev. Lett. 87 (2001) 160601.

[100] J.W. Cherrington and J. D. Christensen, Nucl. Phys. B 813 (2009) 370 [arXiv:0808.3624 [hep-lat]].

[101] J.W. Cherrington, Nucl. Phys. B 794 (2008) 195 [arXiv:0710.0323 [hep-lat]].

[102] J.W. Cherrington, D. Christensen, I. Khavkine, Phys. Rev. D 76 (2007) 094503 [arXiv:0705.2629].

[103] R. Anishetty and H.S. Sharatchandra, Phys. Rev. Lett. 65 (1990) 813.

[104] P. de Forcrand, J. Langelage, O. Philipsen and W. Unger, arXiv:1312.0589 [hep-lat].

[105] J. Langelage, M. Neuman and O. Philipsen, arXiv:1311.4409 [hep-lat].

[106] M. Fromm, J. Langelage, S. Lottini, M. Neuman, O. Philipsen, Phys. Rev. Lett. 110 (2013) 122001 [arXiv:1207.3005 [hep-lat]].

[107] M. Fromm, J. Langelage, S. Lottini, O. Philipsen, JHEP 1201 (2012) 042. [arXiv:1111.4953].

[108] J. Langelage and O. Philipsen, JHEP 1001 (2010) 089 [arXiv:0911.2577 [hep-lat]].

[109] B.H. Wellegehausen, A. Maas, A. Wipf and L. von Smekal, arXiv:1312.5579 [hep-lat].

[110] L. von Smekal, B.H. Wellegehausen, A. Maas and A. Wipf, arXiv:1310.7745 [hep-lat].

[111] T.D. Cohen, Phys. Rev. Lett. 91 (2003) 222001 [hep-ph/0307089].

[112] J. Bloch, Phys. Rev. Lett. 107 (2011) 132002 [arXiv:1103.3467 [hep-lat]].

[113] J. Bloch, F. Bruckmann, M. Kieburg, K. Splittorff, J.J.M. Verbaarschot, Phys. Rev. D 87 (2013) 3, 034510 [arXiv:1211.3990 [hep-lat]].

[114] J. Bloch, Phys. Rev. D 86 (2012) 074505 [arXiv:1205.5500 [hep-lat]].

[115] J. Bloch, F. Bruckmann and T. Wettig, JHEP 1310 (2013) 140 [arXiv:1307.1416 [hep-lat]].

[116] K. Langfeld, B. Lucini, A. Rago, Phys. Rev. Lett. 109 (2012) 111601 [arXiv:1204.3243 [hep-lat]].

[117] K. Langfeld, J. Pawlowski, B. Lucini, A. Rago and R. Pellegrini, arXiv:1310.8231 [hep-lat].

[118] J. Greensite, J.C. Myers and K. Splittorff, JHEP 1310 (2013) 192 [arXiv:1308.6712 [hep-lat]]. 